\title{
Anesthesia Management in Adolescent Bariatric Surgery
}

\author{
Sena Sarıcaoğlu Öktem, Yasemin Akçaalan, Ezgi Erkılıç \\ Anesthesiology and Reanimation Department, Ataturk City Hospital, Ankara, Turkey \\ Email: eerkilic72@yahoo.com
}

How to cite this paper: Öktem, S.S., Akçaalan, Y. and Erkılıç, E. (2021) Anesthesia Management in Adolescent Bariatric Surgery. Open Journal of Anesthesiology, 11, 361-368.

https://doi.org/10.4236/ojanes.2021.1112036

Received: November 5, 2021

Accepted: December 24, 2021

Published: December 27, 2021

Copyright $\odot 2021$ by author(s) and Scientific Research Publishing Inc. This work is licensed under the Creative Commons Attribution International License (CC BY 4.0).

http://creativecommons.org/licenses/by/4.0/

(c) (i) Open Access

\begin{abstract}
Introduction and Purpose: The problem of obesity has started to increase in children and adolescents, similar to adults, due to irregular and unhealthy diet, the limitation of physical activity and genetic factors. Various difficulties and complications may be encountered in the anesthesia management of obese patients. Difficult airway, difficulty in intravenous and intra-arterial intervention, difficulty in positioning the patient, difficulty in finding suitable equipment for the patient, intraoperative and postoperative pulmonary complications, prolonged recovery and difficulty in patient transport are the points to be considered in perioperative management. In our presentation, we wanted to discuss perioperative anesthesia management in adolescent bariatric surgery, which is not performed frequently, with a case study. Case Presentation: A 16-year-old girl with BMI of 44.4 was evaluated for bariatric surgery due to obesity. She had a history of hypertension, sleep apnea and insulin resistance. In her physical examination, her mouth opening was normal, her Mallampati score was 3 , and her neck circumference was $43 \mathrm{~cm}$. After the preoperative difficult intubation preparations were completed, the patient was monitored. The patient was preoxygenated with $100 \%$ oxygen for 3 minutes. The patient with comfortable mask ventilation was intubated with an appropriately sized endotracheal tube. Hemodynamics and oxygen saturation remained stable throughout the surgery. At the end of the surgery, she was extubated without any problem and transferred to the pediatric surgical intensive care unit. The patient was taken to the ward on the 2nd postoperative day and discharged on the 6th day. Discussion and Conclusion: The problem of obesity in adolescents is increasing today, and it is possible to struggle with obesity by making lifestyle changes, teaching children and parents about healthy nutrition, encouraging more physical activity and creating weight control programs. At this point, metabolic and bariatric surgery emerges as a preferred method in overweight, obese adolescents. The anesthesia risks of these surgeries are significantly higher. Postoperative pain management and respiratory physiotherapy should be
\end{abstract}


performed in obese patients. Although prevention of obesity is the mainstay of obesity treatment, bariatric surgery is also a safe and effective treatment method in adolescents.

\section{Keywords}

Anesthesia, Bariatric Surgery, Obesity

\section{Introduction}

Obesity is a chronic disease caused by an increase in fat tissue due to the energy taken in the body being more than that which is spent [1]. Its prevalence has increased worldwide in all age groups in recent years, and it has become a significant public health problem. As in adults, the prevalence of obesity is increasing in children and adolescents due to irregular and unhealthy diet, limited physical activity, and genetic factors. According to the World Health Organization (WHO) 2016 data, in just 40 years, the number of obese school-age children and adolescents has increased by more than 10 times, from 11 million to 124 million. In 2016, more than 340 million children and adolescents, aged 5 - 19, were determined to be overweight or obese. It was seen that the number of obese children under the age of 5 increased to 39 million in 2020 [2]. According to the Turkish Childhood Obesity Research Initiative Study (COSI-TUR)-2016 data, between 2013 and 2016, obesity in Turkey in 2nd-grade children, aged 7 - 8 years, increased by $19.3 \%$, and this increase, especially in girls, was alarming. The WHO generated a policy under the slogan "The Global Action Plan on Physical Activity 2018-2030: More Active People for a Healthier World", aiming for 2030, to prevent an increase in obesity in children, and this policy has begun to be implemented by WHO member countries [2]. Likewise, the "Action Plan for the Prevention of Childhood Obesity", prepared by the Turkish Ministry of Health, General Directorate of Public Health, started to be implemented in 2019 and will continue until 2023.

Obesity is often defined by the Body Mass Index (BMI). The BMI is the weight ratio to the square of the height $\left(\mathrm{kg} / \mathrm{m}^{2}\right)$ [3]. Percentile curves are also used in children and adolescents. Children over the age of two are considered overweight if they are above the 85 th percentile, obese if they are above the 95th percentile, and morbidly obese if they are above the 99th percentile. Moreover, $85 \%$ of children's ages under two years are defined as overweight and not obese depending on the required weight of that month [4]. Although its etiology is not entirely clear, genetic and environmental factors play a significant role. It is a complex, multifactorial disease that is characterized by behavioral, endocrine, and metabolic changes. Nutritional history, lifestyle and physical activity, psychosocial status, medication (antiepileptics, steroids, antidepressants, etc.), sleep problems, existing diseases, previous operations, and family history are among the impor- 
tant risk factors for obesity. Due to multiple etiological factors, diet and lifestyle changes used in the treatment of obesity may not be effective for every patient. In order to see long-term success, patients need to make permanent changes in their lifestyles. In recent years, a lot of preparations have been approved and started to be used for the medical treatment of obesity. However, the long-term safety information and efficacy of these therapeutic agents are limited. Because of these reasons, the surgical treatment of obesity is getting more and more attention day by day.

Adolescent obesity causes serious health problems in both adolescence and adulthood. Cardiovascular, respiratory (asthma, obstructive sleep apnea syndrome (OSAS), etc.), and metabolic diseases, orthopedics, urinary system, dermatological, gastrointestinal, neurological system, psychosocial problems, cancer types, and problems related to puberty (adrenarche, early puberty, etc.) may often coexist [4].

It is possible to encounter various difficulties and complications in the anesthesia management of obese patients. Difficult airway, difficulty in intravenous and intraarterial intervention, positioning of the patient and finding convenient equipment, intraoperative and postoperative pulmonary complications, prolonged recovery, and difficult patient transport are the most common problems in anesthesia management of obese patients.

\section{Case}

A 16-year-old, 136-kg, 175-cm-tall, female patient was scheduled for laparoscopic sleeve gastrectomy surgery, and had a BMI of 44.4, Hypertension (HT), sleep apnea, and insulin resistance. She underwent adenoidectomy at the age of 9 . There were no complications. The patient, who applied to an external center for rapid weight gain 5 years previously, was recommended a diet and follow-up. She lost $10 \mathrm{~kg}$ with the diet, and then her weight loss decreased. After she stopped the diet, she gained $20 \mathrm{~kg}$. Metformin oral tablets had been administered 3 years prior due to high insulin resistance, in addition to enapril tablets due to the diagnosis of hypertension. The patient, for whom bariatric surgery due to obesity was planned, had applied to our hospital's pediatric surgery clinic.

When the patient was first brought to the operating table, her general condition was good. She was conscious, oriented, cooperative, spontaneous breathing was comfortable, and she did not need oxygen support. Systemic physical examination was normal. The patient's mouth opening was normal, her mallampati was $3 \mathrm{~cm}$ and her neck circumference was $43 \mathrm{~cm}$. The cardiology department was consulted in the preoperative evaluation of the patient. Echo evaluation was routine, and the cardiology department found no inconvenience in administering the anesthesia. Enapril and metformin were discontinued 1 day before surgery. In her preoperative blood, her hemoglobin $(\mathrm{Hb})$ was $12.1 \mathrm{~g} / \mathrm{dL}$, hematocrit (Hct) was $39.2 \%$, Platelet Count (PLT) was 291.000, hemoglobin A1c (HbA1C) was 5.5\%, fasting blood sugar was $85 \mathrm{mg} / \mathrm{dL}$, postprandial insulin was $129.99 \mathrm{Uu} / \mathrm{mL}$, and her TFT, 
fasting insulin, and cortisol levels were normal.

The patient's ECG, oxygen saturation, and noninvasive blood pressure were monitored on the operating table. The patient's 20-gauge vascular access from dorsum of her right hand was checked in the ward, and $1000 \mathrm{cc}$ of isotonic fluid was inserted. Preparations were made for difficult intubation. A video laryngoscope was prepared in the room (Karl Storz-endoscope c-mac 8403 ZX). Airway, Laryngeal Mask Airway (LMA), intubation tubes, and masks of various sizes were set out. The patient was in the supine position. The external auditory canal and sternal notch were brought to the same plane by supporting the head and the lower neck. The operating table was placed in a ramp position. The patient was preoxygenated with $100 \%$ oxygen for $3 \mathrm{~min}$. During induction, $60 \mathrm{mg}$ of arrhythmic, $200 \mathrm{mg}$ of propofol, $100 \mathrm{mcg}$ of fentanyl, $80 \mathrm{mg}$ of rocuronium were administered Intravenously (IV). The patient was ventilated with a medium-sized mask when her spontaneous breathing stopped. The mask comforted the ventilation. After the patient was relaxed entirely, direct laryngoscopy was performed, and the patient was intubated with a Machintosh No. 3 blade and a No. 7.0 cuffed tube simultaneously. The Cormack-Lahen score was 2 , and it was not considered as a difficult intubation. Moreover, $2 \%$ sevoflurane and a remifentanil infusion of 0.1 $0.2 \mathrm{mcg} / \mathrm{kg} / \mathrm{min}$ was administered for anesthesia maintenance. Following this, 18gauge vascular access was opened from the dorsum of her left hand, and 16-gauge vascular access was made on the inside of her right wrist. A catheter was inserted for urine output monitoring. The surgical procedure was started. In addition to $1000 \mathrm{cc}$ of isotonic fluid, $500 \mathrm{cc}$ of isolyt-s was inserted. The abdomen was inflated with $\mathrm{CO}_{2}$ by the surgeon until the pressure reached $12 \mathrm{mmHg}$. Ventilation was provided in volume-controlled mode with a $7-\mathrm{mL} / \mathrm{kg}$ tidal volume and $\mathrm{fio}_{2}$ of 0.5 according to the ideal weight. Peak pressures were regular, and there was no increase. The patient's hemodynamics and saturation remained stable throughout the surgery. A total of $2500 \mathrm{~mL}$ of crystalloid fluid was given. The urine output was $1050 \mathrm{~mL}$. An additional dose of $30 \mathrm{mg}$ of rocuronium was administered at intervals of $10 \mathrm{mg} / \mathrm{h}$. Thirty minutes before the end of the surgical procedure, 100 $\mathrm{mg}$ of tramadol and $4 \mathrm{mg}$ of ondansetron were administered IV. The surgical procedure lasted $4 \mathrm{~h}$ and $15 \mathrm{~min}$. Additionally, $2 \mathrm{mg} / \mathrm{mL}$ of tramadol and IV patient controlled analgesia (PCA) were prepared for postoperative analgesia. The PCA was adjusted to a $20-\mathrm{mg} / \mathrm{h}$ basal infusion, $10-\mathrm{mg}$ bolus dose, lockout time of $20 \mathrm{~min}, 4 \mathrm{~h}$ limit of $100 \mathrm{mg}$, and was inserted at the end of surgery. After all of the anesthetics had been turned off during the extubation phase, the patient began to be ventilated with $100 \%$ oxygen. Next, $200 \mathrm{mg}$ of sugammadex was administered IV. When the tidal volume was adequate, the patient was extubated and taken to the recovery room consciously and cooperatively. Her vitals were stable, and her oxygen-free $\mathrm{SpO}_{2}$ was 98 . The patient was transferred to the postoperative pediatric surgical intensive care unit. Pain control was achieved with PCA IV in the first $24 \mathrm{~h}$ postoperatively. The patient was taken to the service on day 2 postoperatively and discharged on day 6 postoperatively. 


\section{Discussion}

The problem of obesity in adolescents is increasing daily. It is possible to combat obesity by making lifestyle changes, teaching children and parents about healthy nutrition, encouraging more physical activity, and creating weight control programs. Sometimes, weight control is achieved successfully with lifestyle changes in overweight children, while gaining weight again is observed in the long-term results. At this point, metabolic and bariatric surgery emerges as a preferred method in overweight, obese, and adolescents with obesity-related diseases. Although the prevention of obesity is the mainstay of obesity treatment, bariatric surgery is also a safe and effective treatment method in adolescents [3].

Bariatric surgery contraindications:

1) Presence of an untreated endocrine disease that causes obesity (such as Cushing's, hypothyroidism, insulinoma);

2) Having an untreated eating disorder (such as bulimia nervosa);

3) Presence of untreated major depression or psychosis;

4) Presence of severe coagulopathy;

5) Alcohol or substance abuse;

6) Inability to comply with dietary recommendations such as lifelong vitamin replacement or calorie-restrictive diet;

7) Having cancer disease;

8) Severe gastroesophageal reflux disease (GERD) (especially for sleeve gastrectomy);

9) Portal hypertension;

10) Gastric bypass surgery in patients with Crohn's disease.

In the preoperative evaluation in adolescent bariatric surgery, the BMI, heart rate, blood pressure, blood sugar, oxygen saturation (in room air) should be optimized. OSAS risk factors, drugs used, evaluation and management of preoperative anxiety, informing the family, and assessment of venous access and risks is essential.

In risk assessment, according to the American Society of Anesthesiologists (ASA) classification, patients with a high BMI are included in the ASA II risk group, even if they do not have any co-morbid diseases. Morbidly obese patients are evaluated directly as ASA III [4].

Much obesity-related co-morbidities are seen in adolescents. Cardiovascular diseases, type 2 diabetes, OSAS, non-alcoholic fatty liver, orthopedic problems (slipped femoral epiphysis, tibia vara), and gastroesophageal reflux are the most common diseases associated with obesity in adolescents [3]. There are also genetic syndromes accompanying obesity. Perioperative anesthetic risks have also increased due to these diseases.

Detailed physical examination, especially regarding difficult airway risk assessment, should be done (mouth opening, head, neck, and jaw movements, mallampati score should be evaluated). Drugs and doses that can cause respiratory depression should be avoided in premedication. Midazolam is one of the most commonly used 
drugs in premedication. It is recommended to calculate more than the ideal weight to avoid the effects of respiratory depression [4]. Obese patients may also experience difficulties in monitorization due to excess adipose tissue. It can be challenging to find the appropriate size blood pressure cuff for noninvasive blood pressure measurement.

Thicker subcutaneous adipose tissue complicates intraarterial and intravenous access in obese adolescents. On the volar side of the hand, the veins may be easier to see and cannulate due to their being less adipose tissue in the tendons. Ultrasound and devices that make the veins visible can be used.

Inhalation and intravenous methods can be used for anesthesia induction. Sevoflurane is preferred for inhalation anesthesia because it causes less airway irritation. In general, the volume of distribution of lipophilic drugs from intravenous anesthetic agents increases, while that of hydrophilic agents does not change [5].

Precautions should be taken regarding difficult airway, various sizes of airway, mask, LMA, intubation tube, laryngoscope blades, and video laryngoscope, and fiberoscope should be available. The external auditory canal and sternal notch should be brought to the same plane by positioning the head appropriately. The table should be in the ramp position. Preoxygenation should be performed by breathing 4 deep breaths for at least $3 \mathrm{~min}$ or $30 \mathrm{~s}$ with $100 \%$ oxygen. Difficult ventilation and difficult intubation should be considered, and awake intubation with fiberoptic should be kept in mind. Sugammadex should be available as a precaution in the event that there is an inability to ventilate, and induction with short-acting general anesthetics should be preferred.

No difference was detected during the use of the volume and pressure control when using intraoperative mechanical ventilators. However, it has been observed that pressure-controlled ventilation at a certain peak pressure provides higher tidal volumes than volume-controlled ventilation. Controlled ventilation with Positive End-Expiratory Pressure (PEEP) is helpful to prevent atelectasis, but hypotension may develop, and this requires attention. In order not to develop resorption atelectasis, the $\mathrm{FiO}_{2}$ should be kept between 0.4 and 0.8 , including the pre-extubation period [6].

The amount of fluid to be given in the intraoperative fluid management is calculated according to the ideal body weight. Attention should be paid to surgical fluid losses, monitoring urine output, and avoiding hypo-hyperglycemia. Fluid supplementation should be performed considering the increased risk of rhabdomyolysis in laparoscopic surgical procedures in obese patients.

Particular attention should be paid to the positions during surgery. Adipose tissue is avascular, so pressure ulcers may occur. Soft pads should support the bony prominences. Postoperative pulmonary complications are common in obese patients. These complications include atelectasis, pneumonia, laryngospasm, respiratory distress, ventilation/perfusion mismatch, and increased need for reintubation. Lung dynamics may change due to increased intra-abdominal pressure. This oc- 
curs in the supine, lithotomy, and most often, Trendelenburg positions. As a result, the diaphragm is pushed up, and the tidal volume, functional residual capacity, total lung capacity, compliance, inspiratory capacity decrease, and ventilation/perfusion impairment develops. Lateral decubitus and prone position can be tolerated more easily. Extubation may have a higher risk than intubation. After the patient is put into a sitting position at $30^{\circ}-45^{\circ}$, it is necessary to wait until the effect of the neuromuscular blockers is completely reversed and the patient is fully awake [7]. Moreover, reintubation should be prepared for.

Postoperative aggressive pain management and respiratory physiotherapy should be performed in obese patients. Thoracic epidural analgesia is safe and effective in postoperative pain. Intravenous PCA, local anesthetic infiltration, paracetamol, nonsteroidal antiinflammatory medication, and dexmedetomidine can be used. Spontaneous Venous Thromboembolism (VTE) is known to be twice as common in obese patients when compared to non-obese patients. Early mobilization and compression stockings can prevent VTE [8].

\section{Conclusions}

Obesity in adolescents is increasing rapidly globally, as well as in Turkey, due to multifactorial reasons, such as a sedentary lifestyle, unhealthy diet, and genetic factors. Obesity poses significant psychological, social, and health problems in adolescents. It brings with its lots of co-morbidities. For these reasons, obesity treatment has gained importance. Bariatric surgery methods used in treating obesity in adults are now being used frequently and safely in pediatric patients.

The anesthetic risks of these surgeries are significantly higher. The risk of difficult mask ventilation, difficult intubation, postoperative atelectasis and thromboembolism is high. None of these were found in the case herein. However, these risks and complications must be considered.

\section{Conflicts of Interest}

The author declares no conflicts of interest regarding the publication of this paper.

\section{References}

[1] Dilek OZCELİK ERSU (2016) Determination of the Relationship between Obesity and Nutritional Status and Kidney and Liver Functions in Children and Adolescents. İzmir Katip Celebi University Faculty of Health Sciences Journal, 1, 13-19.

[2] WHO: Obesity and Overweight. https://www.who.int/news-room/fact-sheets/detail/obesity-and-overweight

[3] Sugerman, H.J., Sugerman, E.L., DeMaria, E.J., Kellum, J.M., Kennedy, C., Mowery, Y. and Wolfe, L.G. (2003) Bariatric Surgery for Severely Obese Adolescents. Journal of Gastrointestinal Surgery, 7, 102-108. https://doi.org/10.1016/S1091-255X(02)00125-7

[4] Erkılıç, E. (2020) Anesthesia in Obese Children. Balıkesir Medical Journal, 4, 40-51. https://doi.org/10.33716/bmedj.678177 
[5] SOBA: Guidelines for the Patient with BMI $>35$.

https://www.sobauk.co.uk/guidelines-1 available date 18.08.2021

[6] Souza, G.M.C., Santos, G.M., Zimpel, S.A. and Melnik, T. (2020) Intraoperative Ventilation Strategies for Obese Patients Undergoing Bariatric Surgery: Systematic Review and Meta-Analysis. BMC Anesthesiology, 20, Article No. 36.

https://doi.org/10.1186/s12871-020-0936-y

[7] Marjanovic, V., Budic, I., Golubovic, M. and Breschan, C. (2021) Perioperative Respiratory Adverse Events during Ambulatory Anesthesia in Obese Children. Irish Journal of Medical Science. https://doi.org/10.1007/s11845-021-02659-3

[8] Sandblad, K.G., Jern, S., Åberg, M., Robertson, J., Torén, K., Lindgren, M., Adiels, M., Hansson, P.O. and Rosengren, A. (2020) Obesity in Adolescent Men Increases the Risk of Venous Thromboembolism in Adult Life. Journal of Internal Medicine, 734-745. https://doi.org/10.1111/joim.13044 